\title{
Identification and in vivo characterization of a brain-penetrating nanobody
}

\author{
Y Wouters $^{1,2}$, T Jaspers $^{1,2}$, B De Strooper ${ }^{1,2,3^{*}}$ and M Dewilde ${ }^{1,2,4,5^{*}}$ (D)
}

\begin{abstract}
Background: Preclinical models to determine blood to brain transport ability of therapeutics are often ambiguous. In this study a method is developed that relies on CNS target-engagement and is able to rank brain-penetrating capacities. This method led to the discovery of an anti-transferrin receptor nanobody that is able to deliver a biologically active peptide to the brain via receptor-mediated transcytosis.

Methods: Various nanobodies against the mouse transferrin receptor were fused to neurotensin and injected peripherally in mice. Neurotensin is a neuropeptide that causes hypothermia when present in the brain but is unable to reach the brain from the periphery. Continuous body temperature measurements were used as a readout for brain penetration of nanobody-neurotensin fusions after its peripheral administration. Full temperature curves were analyzed using two-way ANOVA with Dunnett multiple comparisons tests.

Results: One anti-transferrin receptor nanobody coupled to neurotensin elicited a drop in body temperature following intravenous injection. Epitope binning indicated that this nanobody bound a distinct transferrin receptor epitope compared to the non-crossing nanobodies. This brain-penetrating nanobody was used to characterize the in vivo hypothermia model. The hypothermic effect caused by neurotensin is dose-dependent and could be used to directly compare peripheral administration routes and various nanobodies in terms of brain exposure.
\end{abstract}

Conclusion: This method led to the discovery of an anti-transferrin receptor nanobody that can reach the brain via receptor-mediated transcytosis after peripheral administration. This method could be used to assess novel proteins for brain-penetrating capabilities using a target-engaging readout.

Keywords: Nanobody, VHH, Transferrin receptor, Neurotensin, Blood-brain barrier, Receptor-mediated transcytosis

\section{Background}

Delivery of biologics to the central nervous system (CNS) has been a major challenge. This is partly due to the fact that the CNS is physically separated from the periphery by several barriers, including the blood-brain barrier (BBB), a monolayer of endothelial cells supported by astrocytes and pericytes [1]. It is estimated that only $0.1 \%$ of circulating macromolecules is able to reach the brain

\footnotetext{
*Correspondence: bart.destrooper@kuleuven.vib.be; maarten. dewilde@kuleuven.be

${ }^{1}$ VIB Center for Brain \& Disease Research, Campus Gasthuisberg O\&N4, Herestraat 49, box 602, B-3000 Leuven, Belgium

Full list of author information is available at the end of the article
}

parenchyma, which severely limits the use of biologics to treat CNS-related diseases [2].

Transcytosis pathways involved in the delivery of essential nutrients have been explored for delivery of drugs to the brain. Nutrients are able to cross the BBB via specific receptors expressed on the luminal side of brain endothelial cells via receptor-mediated transcytosis (RMT). So-called Trojan Horse approaches exploit this mechanism. Therapeutic biologics are coupled to receptor-targeting entities such as peptides or antibodies binding to these nutrient receptors to shuttle them to the brain [3, 4]. Regardless intense research of RMT [5] during the past 30 years, only two drug candidates successfully completed phase 1-2 clinical trials [6, 7]. Moreover, 
currently there are no therapeutic proteins approved for clinical use that cross the BBB to exert their effect [8].

Nanobodies (also called single-domain antibodies or VHHs) could be an interesting addition to the existing Trojan Horse delivery methods. Nanobodies are derived from the variable domain of the heavy-chain-only antibodies found in camelids $[9,10]$. They recognize antigens with similar affinities and specificity as monoclonal antibodies and can be easily fused to a wide variety of compounds [11-15]. Nanobodies have been used in various fields, ranging from therapeutic to biochemistry applications [10, 16-18].

Many reports have claimed access of nanobodies to the brain. Due to their small size and often cationic charge, nanobodies are able to fuse with the negatively charged cell membrane which can lead to brain uptake via adsorptive-mediated transcytosis [19]. This mechanism entirely relies on the charge of the brain-penetrating entity. Fusion to a therapeutic compound changes this charge which then alters crossing efficiency [20-22]. Since small changes or fusions of various entities to nanobodies that reach the brain via adsorptive-mediated transcytosis can alter their crossing capabilities, such nanobodies are not suitable as a Trojan Horse delivery systems. Instead, nanobodies that utilize RMT to deliver drugs to the CNS could be more successful Trojan Horses.

One of the most investigated RMT targets is the transferrin receptor (TfR), which is highly expressed on brain endothelial cells $[5,23]$. Anti-TfR monoclonal antibodies deliver therapeutics to the brain in rats [24,25], mice [26, 27], monkeys [4] and humans [7]. Also Tfr antibody-fragments, such as single-chain variable fragments [28-30], Fab fragments [31] or dual variable domain immunoglobulins [32], are able to facilitate blood to brain transport. Very recently, two studies have showed that engineering the Fc fragment of a monoclonal antibody to target the TfR resulted in more brain uptake of various therapeutic proteins [33, 34]. This previous research opens the door for the discovery of brain-penetrating, anti-TfR nanobodies. In order to select potential Trojan Horse nanobodies (meaning nanobodies reaching the brain via RMT and not by adsorptive-mediated transcytosis), unequivocal preclinical evidence for transfer to the brain has to be delivered.

A reliable method to demonstrate CNS target-engagement is inhibition of beta-secretase 1 (BACE1). BACE1 is an enzyme that cleaves the amyloid precursor protein (APP) in neuronal endosomes leading to the generation of amyloid- $\beta$ peptides $(\mathrm{A} \beta)[35,36]$. Here, brain-penetrating moieties fused to a BACE1 inhibiting entity are intravenously injected into animal species, after which the brains are harvested and homogenized. A decrease in central $A \beta$ levels, measured by ELISA, indicates BBB crossing [33, 37-40]. While this proves good evidence for functional brain targeting this method requires for each measurement point the use of multiple mice [41]. Moreover the further processing of brain extracts and ELISAs are time consuming and expensive, prohibiting large screening efforts.

In order to improve the robustness of preclinical in vivo CNS research and reduce the number of animals needed for proof-of-concept, a method is needed that demonstrates brain uptake by target engagement, is unambiguous with regard to brain target, and, finally, allows for reuse of laboratory animals. Here, we explore whether it is possible to use a nanobody to reach the brain using new nanobodies raised against the mouse Transferrin receptor TfR (mTfR), a known receptor-mediated transcytosis target. As a readout, we coupled the nanobodies to NT, a neuropeptide that elicits hypothermia after binding to the NT receptor (NTSR1) expressed in the CNS [42]. Since this receptor is a G-protein-coupled receptor (GPCR) located in the cell membrane of hypothalamic neurons [43], it can interact with molecules in the interstitial fluid. In contrast to intracerebroventricular administered NT, intravenously administered NT is not able to elicit a hypothermic response [44]. Therefore, the observed hypothermic effect after intravenous injection of nanobody-NT fusions is direct evidence of BBB transport facilitated by that particular nanobody. Moreover, this method assesses the brain penetrating capabilities of the generated nanobodies after a single IV injection and without the need to sacrifice the animal, making it possible to use the animal multiple times. The developed method led to the discovery of the first nanobodies that can deliver a cargo (NT) to the brain via the TfR.

\section{Materials and methods Nanobody library generation}

Nanobodies targeting the mouse transferrin receptor were obtained in collaboration with the VIB Nanobody Core. A llama was immunized with the extracellular domain of the mouse transferrin receptor $(50741-\mathrm{M} 07 \mathrm{H}-$ 100 , Sino Biological) by subcutaneous injections on days $0,7,14,21,28$ and 35 . The first injection contained $100 \mu \mathrm{g}$ protein followed by five injections with $70 \mu \mathrm{g}$ protein each. On day 40 a blood sample of $100 \mathrm{ml}$ was collected and peripheral blood lymphocytes were isolated. The nanobody library was cloned into a phagemid vector as previously prescribed [45]. Briefly, total RNA from peripheral blood lymphocytes was used as template for first strand cDNA synthesis with oligodT primer. This cDNA was used to amplify the nanobody-encoding open reading frames by PCR, digested with PstI and NotI, and cloned into the phagemid vector pMECS. The library 
was transformed into electro-competent E.coli TG1 cells, which resulted in $10^{8}$ independent transformants, of which $85 \%$ contained the vector with a right insert size.

\section{Isolation of anti-mTfR nanobodies}

To select anti-mTfR nanobodies, two rounds of in solution selections were performed with 100 and $50 \mathrm{nM}$ biotinylated mTfR (50741-M07H-100, Sino Biological), respectively. After the second round the library was subcloned into an expression vector (pBDS100, a modified pHEN6 vector with an OmpA signal peptide and a C-terminal 3xFlag/6xHis tag) [46]. The expression library was used to transform TG1 E.coli after which nanobodies were expressed from single colonies. These nanobodies were screened for direct binding to the biotinylated mTfR using the AlphaScreen Histidine Detection Kit (6760619 M, Perkin Elmer). The hits were sequenced and clustered according to sequence homology. One representative of each sequence cluster was recloned into the NT vector (pBDS100 with C-terminal NT), expressed and purified following the protocol by Pardon et al. [45]. In total, 7 nanobodies were successfully recloned and expressed.

\section{Generation of affinity variants}

Single amino acid substitutions in the CDR3 region of Nb62 were generated following the protocol of Kille et al. [47]. In short, 13 separate PCRs were performed using the Phusion High-Fidelity PCR Kit (F553S, Thermo Scientific) and purified via agarose gel electrophoresis and a QIAquick Gel Extraction Kit (28704, Qiagen). The methylated template DNA constructs were removed by $D p n I$ digestion and the products were purified using QIAquick PCR Purification Kit (28104, Qiagen). Next, the plasmids were mixed in equimolar amounts and transformed into TG1 E.coli. Screening of single colonies was performed as described above.

\section{In vitro binding \\ Bio-layer interferometry}

Binding of the purified nanobodies to various forms of the transferrin receptor was assessed using an Octet RED96 (Forté Bio/Molecular Devices). Initially, streptavidin (SA) biosensor tips (18-5020, Forté Bio/Molecular Devices) were pre-wet for minimally $10 \mathrm{~min}$ in $1 \mathrm{xPBS}$, after which they were dipped in biotinylated TfR $(1 \mu \mathrm{g} /$ $\mathrm{ml}$ in $1 \mathrm{xPBS})$. Next, the tips were sequentially submerged in baseline wells ( $1 \times \mathrm{PBS})$, dissociation wells $(1 \mathrm{xPBS})$, nanobodies (100 nM in 1xPBS) and dissociation wells.
Sensorgrams were generated using the Forté Bio Octet RED analysis software (Forté Bio/Molecular Devices).

\section{ELISA}

Nunc maxisorp ${ }^{\mathrm{TM}}$ 96-well plates (44-2404-21, Thermo Fisher) were coated with $0.1 \mu \mathrm{g}$ of mTfR in $100 \mu \mathrm{l}$ PBS per well and incubated overnight at $4{ }^{\circ} \mathrm{C}$. The next day, plates were washed 5 times with $200 \mu \mathrm{l}$ PBS $(0,05 \%$ tween-20) and blocked with $150 \mu \mathrm{l}$ PBS (0,1\% casein) for $1 \mathrm{~h}$ at room temperature. Then, $50 \mu \mathrm{l}$ of dilution series from $10 \mu \mathrm{M}$ to $0,1 \mathrm{nM}$ in blocking buffer per nanobody were added and incubated for $1 \mathrm{~h}$ at room temperature, followed by 5 washes. Subsequently, $50 \mu \mathrm{l}$ of mouse antiFLAG M2 monoclonal antibody (F3165, Sigma, 1 in 20.000 diluted in PBS/casein) was added for $1 \mathrm{~h}$ at room temperature, followed by 5 washes and $50 \mu$ of horseradish peroxidase-conjugated goat anti-mouse antibody (P0447, DAKO, 1 in 20.000 diluted in PBS/casein) for $1 \mathrm{~h}$ at room temperature. Next, 5 washes were performed and the reaction was developed using $100 \mu \mathrm{l}$ of developing solution $(10 \mathrm{~mL}$ sodium acetate $(\mathrm{pH} 4.9), 100 \mu \mathrm{l}$ 3,3',5,5'-Tetramethylbenzidine, $10 \mu \mathrm{l}$ hydrogen peroxide) and stopped upon blue color formation using $100 \mu \mathrm{l}$ sulfuric acid $(2 \mathrm{~N})$. Absorbance at $450 \mathrm{~nm}$ was measured using an EnVision ${ }^{\circledR}$ multimode plate reader (Perkin Elmer).

\section{In vivo binding \\ Animals}

All animal experiments were conducted according to protocols approved by the local Ethical Committee of Laboratory Animals of the KU Leuven (governmental license LA1210579, ECD project number 040/2016) following governmental and EU guidelines. The in vivo experiments were performed using both male and female mice ages $2-6$ months. The sample size $(n=3)$ was calculated by a continuous endpoint, two independent sample groups using the following parameters: mean $\Delta \mathrm{T}$ group $1=0{ }^{\circ} \mathrm{C}$, standard deviation on $\Delta \mathrm{T} \pm 0.5{ }^{\circ} \mathrm{C}$, mean $\Delta \mathrm{T}$ group $2=2.5^{\circ} \mathrm{C}, p$ value $\leq 0.05$ and power $=0,8$.

\section{Anipill ${ }^{\circledR}$ implantation}

To automatically measure body temperature of socially housed mice, the Anipill ${ }^{\circledR}$ (BodyCap) system was implanted in TLR4 ${ }^{-1-}$ mice. These mice are resistant to endotoxins, which would prevent potential BBB opening by residual endotoxins. TLR $4^{-1-}$ mice were injected with buprenorphine $(0,05 \mathrm{mg} / \mathrm{kg}, \mathrm{SC})$ an hour before Anipill ${ }^{\circledR}$ implantation, followed by lidocaine $(6 \mathrm{mg} /$ $\mathrm{kg}$, SC under the scalp) as local analgesia five minutes before implantation. The mice were induced with $5 \%$ isoflurane and were placed in the stereotactic frame 
with 1-2\% isoflurane. The abdomen was opened and the Anipill ${ }^{\circledR}$ was implanted. Next, the muscle layer was sutured with resorbable sutures and the skin was closed with surgical staples. Then, $500 \mu \mathrm{l}$ of saline was injected subcutaneously and the animals were allowed to recovery under a heating lamp, followed by an additional injection of buprenorphine $(0.1 \mathrm{mg} / \mathrm{kg}) 6 \mathrm{~h}$ later. The Anipill ${ }^{\circledR}$ implantation was performed at least 1 week prior to any experiment.

\section{ICV injections}

The intracerebroventricular injections were performed as previously described [48] using the following stereotactic coordinates: $\mathrm{AP}-0.1 \mathrm{~mm}, \mathrm{ML}-1.0 \mathrm{~mm}$, and DV-3.0 mm (from the skull). After minimum 1 week of recovery, $2 \mu$ of saline or sample was injected slowly via a Hamilton syringe into the lateral ventricle. Body temperature was monitored every 15 min using the Anipill ${ }^{\circledR}$ system.

\section{IV/IP/SC injections}

For intravenous injections, the mouse was put in a restrainer and the tail was heated in warm water between 42 and $48{ }^{\circ} \mathrm{C}$. Then, nanobodies were injected in the tail vein at volumes between 100 and $180 \mu$ l. For intraperitoneal and subcutaneous injections, the mouse was immobilized and $100 \mu \mathrm{l}$ of the designated nanobody was injected [49]. Body temperature was monitored every 15 min using the Anipill ${ }^{\circledR}$ system.

\section{Results}

\section{Generation and expression of anti-mouse transferrin receptor nanobodies}

In order to find BBB crossing nanobodies, an alpaca was immunized with the extracellular domain of the mouse transferrin receptor. Peripheral blood lymphocytes were isolated, total RNA was extracted and used as template for first strand cDNA synthesis. Next, the nanobodyencoding open reading frames were amplified by a nested polymerase chain reaction, cloned into a phagemid vector and transformed into TG1 E.coli. This yielded a nanobody library with a functional size of about $10^{8}$ independent transformants. To enrich for anti-mTfR-specific nanobodies, two consecutive rounds of phage selection were completed until a 100 -fold increase in binding phages was observed compared to the negative control (no antigen) phage selection by phage titration. Then, the libraries were subcloned into an expression vector, individual clones were picked, expressed and crude extracts were screened using AlphaScreen technology for specific binding to the extracellular domain of the mTfR. In total 282 clones were screened of which 82 nanobodies were able to bind the mTfR. Based on sequence similarity they were classified into 11 different sequence clusters. A representative nanobody was selected for each cluster and 7 nanobodies were successfully re-cloned and expressed as soluble nanobodies with a C-terminal 3XFLAG and a hexahistidine tag followed by neurotensin [50]. Expression was directed to the periplasm after which extraction through osmotic shock was performed. The nanobodies were purified from the resulting extract using immobilized metal-ion affinity chromatography.

\section{In vitro characterization of the anti-mTfR nanobodies}

The protein identity and integrity was confirmed using mass spectrometry (data not shown). Next, binding to the mTfR was confirmed by bio-layer interferometry using the Octet system (Fig. 1a). At the same time binding to the human TfR was also assessed, but none of the nanobodies turned out to be cross-reactive. This lack of cross-reactivity has already been observed for anti-TfR antibodies [51]. We took advantage of this lack of cross reactivity to perform epitope binning. Since currently known brain penetrating monoclonal antibodies bind the apical domain of the TfR [31], a chimeric receptor was generated where the human apical domain was

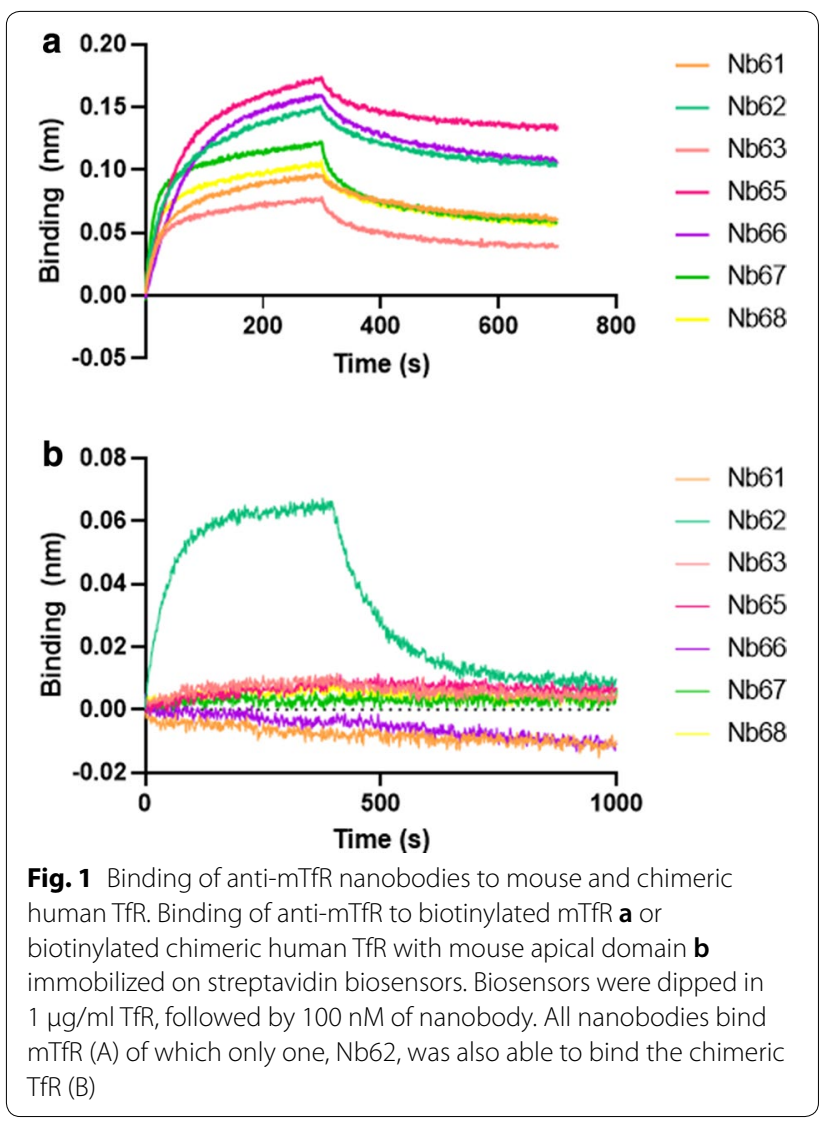


exchanged for the mouse sequence (custom designed, expressed and purified by GenScript Biotech). Again, the Octet system was used to assess binding of the anti-mTfR nanobodies (Fig. 1b). As can be seen, only Nb62 was able to bind the chimeric receptor with mouse apical domain.

\section{In vivo validation of anti-mTfR nanobodies}

In vitro binding however does not mean in vivo crossing. In order to find functionally active anti-mTfR nanobodies, an in vivo screening platform was designed. This platform relies on the NT linked to the nanobodies. NT is a neuropeptide that does not reach the brain parenchyma on its own [50] but lowers the body temperature when it is present in the CNS. If the nanobody-NT fusion would cause a drop in temperature upon IV injection, this would imply that the NT has been transported to the brain by the nanobody. Since nanobodies with a basic isoelectric point $(\mathrm{pI})$ can cross the $\mathrm{BBB}$ via adsorptive-mediated transcytosis $[19,22]$, we calculated the theoretic $\mathrm{pI}$ of all nanobody-NT fusions and took care to incorporate an acidic peptide (AP) between the nanobody and NT sequence so that all overall pIs were acidic, ranging from 5.66 to 7.05 (Fig. 2a). These acidic anti-mTfR nanobodies and a negative control nanobody (raised against green fluorescent protein) were intravenously injected in order to assess their brain-penetrating potential. From the seven injected nanobodies, one (Nb62) was able to elicit a drop in body temperature, which indicated target engagement of NT in the CNS (Fig. 2b). In order to ensure that the lack of decrease in body temperature of the negative control was due to the fact that it did not enter the brain versus a loss of NT function, it was injected directly into the ventricle (Fig. 2c). Here the negative control showed a drop in body temperature, indicating (together with the mass spectrometry data) that the construct contained a functional NT sequence. Next, the brain-penetrating effect of Nb62 was confirmed in three independent mice and showed
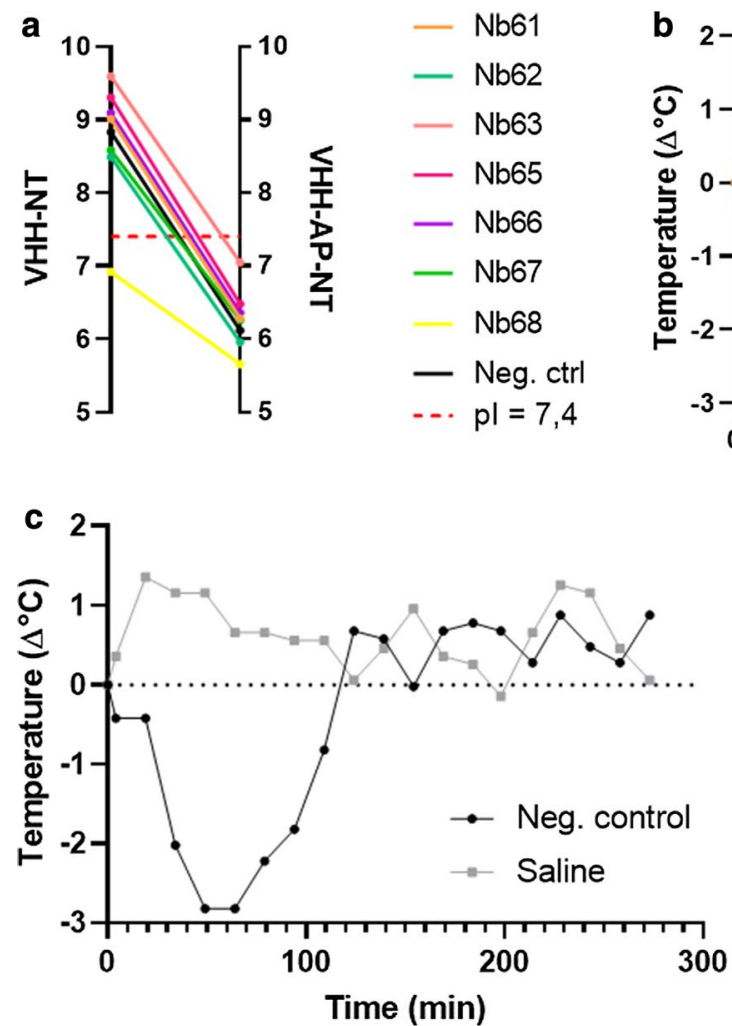
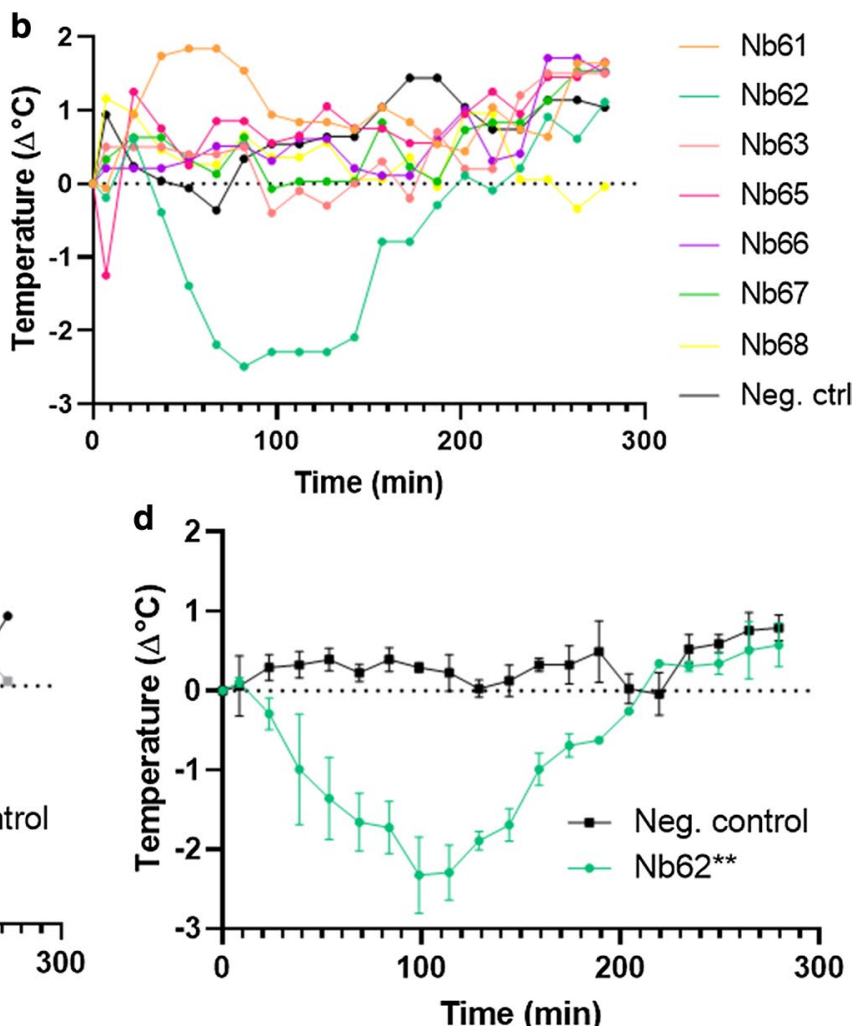

Fig. 2 Evaluation of anti-mTfR nanobodies for receptor-mediated transcytosis. a Theoretical pls of the anti-mTfR nanobodies fused to NT, with or without an incorporated acidic peptide, calculated based on their primary sequence by CLC Main Workbench 8.1. A pl corresponding to no net charge under physiological conditions is indicated by the red dotted line. $\mathbf{b} \mathrm{TLR} 4^{-/-}$mice body temperature measurements after $250 \mathrm{nmol} / \mathrm{kg}$ intravenous injections of the indicated nanobody fused to NT. c TLR4 ${ }^{-/}$mice body temperature measurements after 5 pmol ICV injection of the negative control nanobody fused to NT. $\mathbf{d}$ TLR4 ${ }^{-1-}$ mice body temperature measurements after $250 \mathrm{nmol} / \mathrm{kg}$ IV injections of Nb62 or negative control $(* * p=0.004)$. Bar graphs represent mean $\pm \operatorname{SEM}(n=3$ per group, $p=0.004)$. Statistical test: two-way ANOVA with Dunnett multiple comparisons test compared to negative control nanobody 
a significant decrease in body temperature compared to the negative control (Fig. 2d).

\section{Hypothermic effect by nanobody-NT fusions is dose-dependent}

We next determined whether the method allows dosedependent correlations as this could be used to directly
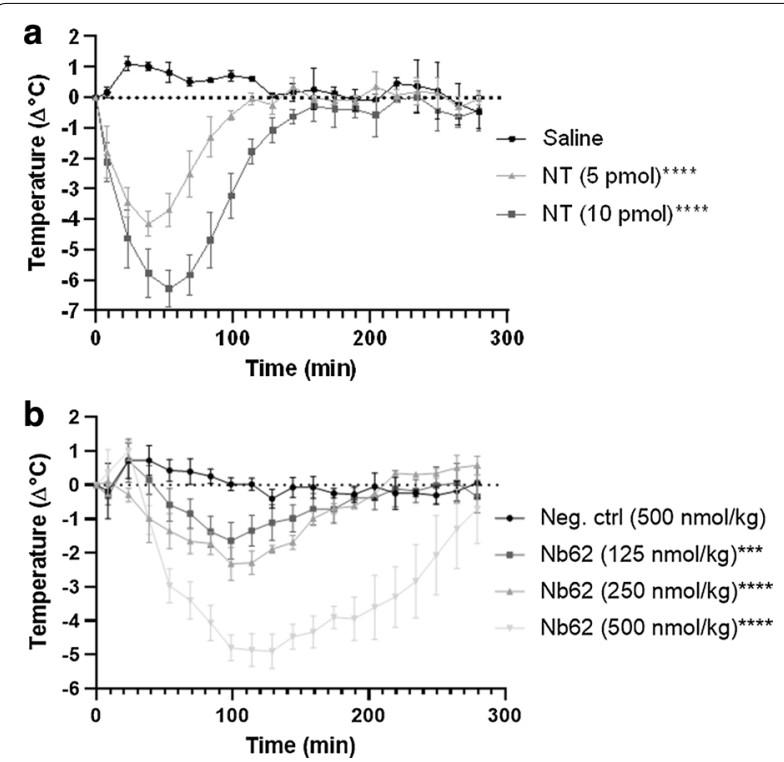

Fig. 3 Evaluation of the dose-response relationship of NT. a TLR4 ${ }^{-1-}$ mice body temperature measurements after ICV injections of the indicated doses of NT or saline $\left({ }^{*} p=0.0249,{ }^{* * * *} p<0.0001\right)$. Bar graphs represent mean \pm SEM $(n=3$ per group for saline and NT (5 pmol) and $\mathrm{n}=2$ for NT (10 pmol)). b TLR4 ${ }^{-1-}$ mice body temperature measurements after intravenous injections of the indicated doses of Nb62 or negative control nanobody fused to NT $\left.{ }^{* * * *} p<0.0001\right)$. Bar graphs represent mean \pm SEM ( $n=3$ per group). Statistical test: two-way ANOVA with Dunnett multiple comparisons test compared to saline or negative control nanobody compare the brain-penetrating efficiency of nanobodies and their modifications. Since NT does not reach the brain by itself, it was injected in the lateral ventricle (ICV) at different doses. As can be seen from Fig. 3a, the drop in body temperature doubles when the dose increases from 5 to $10 \mathrm{pmol}$. This means that the extent of the hypothermic effect is positively correlated with the amount of NT present in the CNS. To determine if this dose-response relationship translates to brain penetration, different doses of Nb62 were injected via the tail vein (Fig. 3b). Again, the decrease in body temperature positively correlates with the amount of injected Nb62. Moreover, the negative control at $500 \mathrm{nmol} / \mathrm{kg}$ did not have a hypothermic effect, indicating that it was not able to reach the CNS. This shows that the hypothermic effect caused by NT can be correlated to the relative amount of nanobody that has actively reached the brain.

\section{NT in vivo screening method can distinguish brain-penetrating efficiency of nanobodies}

A panel of $\mathrm{Nb} 62$ variants was generated by site directed mutagenesis to select the most efficient brain-penetrating nanobody. Their binding curves were generated by ELISA (Fig. 4a). Next, the nanobodies, fused to the catalytic amino acids of NT (8-13), were administered via IV injections at a dose of $250 \mathrm{nmol} / \mathrm{kg}$ and the body temperature of the mice was followed. As can be seen from Fig. $4 \mathrm{~b}$, the mutants give different body temperature profiles, indicating differences in brain uptake.

\section{Delivery route alters brain exposure}

We showed that the NT effect on body temperature is positively correlated to the dose present in the CNS. We next investigated which peripheral delivery route would provide the highest brain exposure. As can be seen from Fig. 5, intraperitoneal (IP) doubled the NT effect in terms
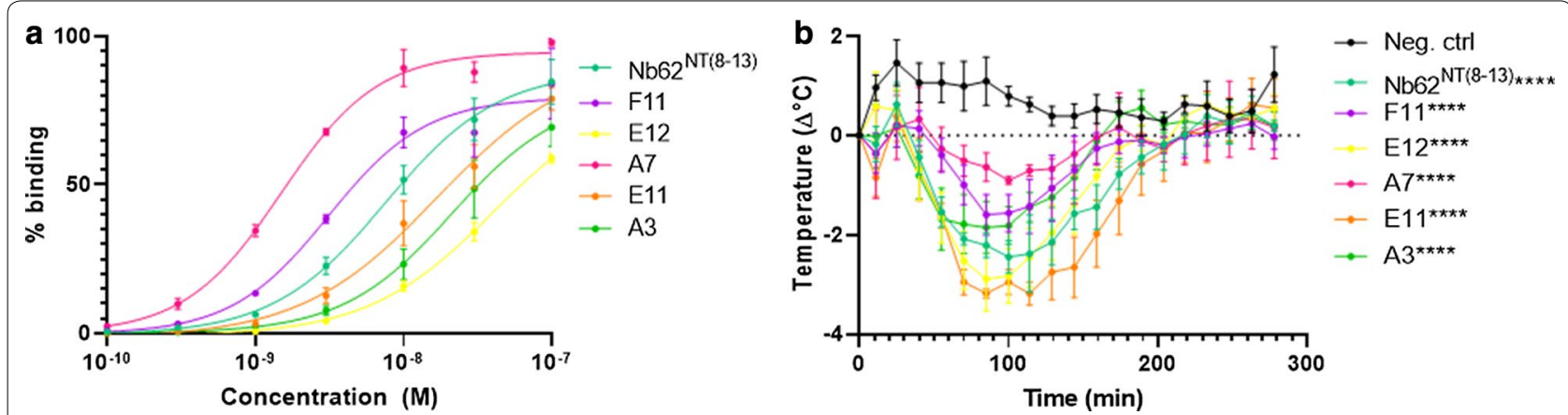

Fig. 4 Brain-penetrating efficiency of Nb62 mutants. a Binding curves of anti-mTfR nanobodies to immobilized mTfR by ELISA ( $n=4)$. Data were fitted by nonlinear regression using the GraphPad Prism 8 software (San Diego, CA, USA). b TLR4 ${ }^{-/}$mice body temperature measurements after IV injections of Nb62 and mutants fused to NT (8-13) at a dose of $250 \mathrm{nmol} / \mathrm{kg}\left({ }^{* * * *} p<0.0001\right)$. Bar graphs represent mean \pm SEM ( $n=3$ per group). Statistical test: two-way ANOVA with Dunnett multiple comparisons test compared to negative control nanobody 


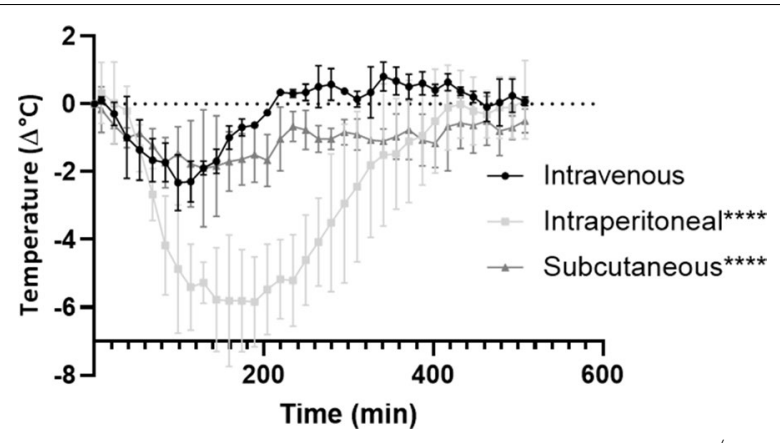

Fig. 5 Effect of delivery route on brain exposure to Nb62. TLR4 ${ }^{-/-}$ mice body temperature measurements after IV, IP or SC injections of $\mathrm{Nb62}$ at a dose of $250 \mathrm{nmol} / \mathrm{kg}\left({ }^{* * *} p<0.001\right)$. Bar graphs represent mean \pm SEM ( $n=3$ per group). Statistical test: two-way ANOVA with Dunnett multiple comparisons test compared to intravenous injection

of temperature drop and duration of the effect compared to IV injection, while the drop in body temperature starts later compared to IV or SC administration. This shows that delivery of $\mathrm{Nb} 62$ intraperitoneally is slower, but leads to more uptake in the brain.

\section{Discussion}

Here, we describe the discovery and characterization of the first anti-mTfR nanobodies that enter the CNS from the periphery via receptor-mediated transcytosis demonstrated by a novel, robust in vivo validation method. This method allows animal re-use and relies on target-engagement by targeting the NTSR 1 expressed on hypothalamic neurons. Nanobodies coupled to NT that are able to penetrate the brain will activate the NTSR1, which causes a drop in body temperature. This hypothermic effect has been described previously as a secondary effect following intravenous administration of ANG2002, which consists of NT fused to a brain-penetrant peptide Angiopep-2, targeting the LDL receptor-related protein-1 [50]. Next to the central hypothermic effect, Demeule et al. also observed in rats a blood pressure reduction following peripheral NT administration. Potentially, this peripheral effect of NT might also be present in mice and could potentially influence the body temperature of the animals. However, no drop in body temperature was observed for our non-BBB crossing controls which were fused to NT. Therefore, the observed temperature drop is centrally mediated and makes NT an ideal tool to validate BBB-crossing of agents like shown by this study. Even though the hypothermic effect of NT has been described before, this is the first time this robust and unambiguous model is used to rank multiple nanobodies in terms of their brain-penetration efficiency. These first anti-mTfR nanobodies are useful tools to study drugs that are targeted to brain targets and are unable to reach the CNS on their own, in a non-invasive way. Moreover, a brain-penetrating nanobody fused to NT itself could be a potential drug candidate in various diseases where body temperature lowering could be beneficial [52-55]. An example is induced hypothermia in acute ischaemic stroke, where a quick, but short duration of hypothermia is beneficial on infarct size [56].

In order to select brain-penetrating nanobodies by receptor-mediated and not adsorptive-mediated transcytosis, an acidic peptide was incorporated between the nanobody and NT sequence, which resulted in only neutral or acidic pI values (Fig. 2a). Next, their brainpenetrating capacities were determined. Out of the 7 nanobodies tested in vivo, only one was able to reach the brain parenchyma. There are several explanations possible why most of the anti-mTfR nanobodies do not cross in vivo. For instance their epitopes might be shielded in vivo by transferrin which has a micromolar plasma concentration and a nanomolar affinity for its receptor [57]. It is known that binding to the apical domain of the TfR can lead to brain uptake of monoclonal antibodies [31]. Here we show this also to be the case for the antimTfR nanobodies, which aligns with other agents reaching the brain via the $\operatorname{TfR}[4,31,58]$.

Next, the hypothermic effect was assessed for dose dependent effects. Different amounts of NT were injected in the lateral ventricles of mice. Higher doses resulted in deeper and longer drops in body temperature (Fig. 3a). Subsequently, Nb62 was injected intravenously with increasing dose. Again, the amount of injected nanobody corresponded with the hypothermic effect. The negative control did not elicit a drop in body temperature at the highest dose, showing there is no passive transport of acidic nanobodies at a dose of $500 \mathrm{nmol} / \mathrm{kg}$. These experiments provide evidence that higher concentrations of brain-penetrating nanobody in the blood leads to more nanobody entering the brain and consequently a bigger NT effect.

Subsequently, we performed a limited structurefunction analysis of Nb62 using our new in vivo screening method to identify more efficient brain-penetrating nanobodies. Here, mutants of $\mathrm{Nb62}$ were generated with different binding affinities, since it is known that affinity for the mTfR affects brain uptake [27, 59]. By using site-directed mutagenesis, single site saturation libraries were generated where each amino acid in the CDR3 region of $\mathrm{Nb62}$, which is most probably involved in antigen binding [60], was substituted to all other amino acids. The nanobodies, fused to the smallest active fragment of neurotensin (NT8-13), were expressed and purified, followed by the generation of binding curves by ELISA (Fig. 4a). The single amino 
acid substitutions resulted in a batch of nanobodies with different binding profiles. Next, the nanobodies were intravenously injected. As can be seen from Fig. 4b, different body temperature drop profiles were observed, indicating differences in brain uptake. Generally, we see that the nanobodies with the strongest binding have the lowest brain uptake. This is in accordance with literature regarding bispecific antibodies targeting the TfR, where it is shown that high affinity monoclonal antibodies are being located to the lysosomes for degradation [61]. In that paper, however, only two monoclonal antibodies were analyzed. All of the analyzed mutants in Fig. 4 induced a significant decrease in body temperature compared to the negative control, indicating they all penetrated the brain to some extent.

Finally, we tested different administration routes for injection of Nb62. IV, IP and SC injections give different PK profiles $[62,63]$, as can be seen from the shapes of the temperature curves in Fig. 5. By comparing the three administration routes, CNS delivery is highest upon IP injection, while a more continuous delivery is reached after SC injection. This is in line with literature where they rely on TfR targeting to deliver an anti-tumor necrosis factor decoy receptor antibody to the brain [63]. These differences in CNS delivery profiles can be contributed to the short plasma half-life of 10-20 min of nanobodies due to fast renal clearance $[64,65]$. An immediate high blood concentration following IV injection might saturate the TfRs present at the $\mathrm{BBB}$, which will deliver the $\mathrm{VHHs}$ to the brain parenchyma. Upon recycling to the luminal side of the plasma membrane, most of the VHH has been cleared from the bloodstream, resulting in the IV profile observed in Fig. 5. IP injection might also saturate the TfRs, but due to the sustained release it is possible for the recycled TfRs to be saturated again. This would lead to a double dose reaching the brain compared to IV, which is indicated by the doubling of the temperature drop and duration of the effect (Fig. 5). SC delivery leads to the lowest plasma concentration [63] which would not saturate the TfRs. However, the sustained release leads to a prolonged brain uptake compared to IV delivery. This is indicated by the plateau of the SC injection profile (Fig. 5).

The short half-life of nanobodies can be interesting for applications such as imaging [22] and PET/CT assessments [66], and can be prolonged for therapeutic applications by fusion to proteins where the nanobody adopts the half-life of the fusion protein, such as the Fc portion of a monoclonal antibody $[67,68]$. Ultimately, next to being a tool to assess the brain-penetrating potential of novel RMT targets, nanobody-NT fusions have the potential to improve the speed of cooling acute stroke patients compared to conventional methods. Here, the short plasma half-life would be beneficial, since an inverse relation between the duration of hypothermia and infarct size is observed [56].

\section{Conclusion}

In conclusion, we have discovered a first set of nanobodies that are able to deliver a payload to the brain via receptor-mediated transcytosis using the transferrin receptor. Simultaneously a novel in vivo platform was set-up to confirm brain penetration of nanobodies in an unambiguous manner. This method is able to directly compare different nanobodies in terms of their brainpenetrating potential. Moreover, the same method was used to show that highest brain uptake was obtained following intraperitoneal delivery. The use of this method could potentially be expanded to other less validated or novel receptor-mediated transcytosis targets to assess their capacity of potential therapeutic delivery towards the brain.

\section{Abbreviations}

Aß: Amyloid- $\beta$; BACE1: Beta-secretase 1; BBB: Blood-brain barrier; CNS: Central nervous system; ELISA: Enzyme-linked immunosorbent assay; ICV: Intracerebroventricular; IP: Intraperitoneal; IV: Intravenous; NT: Neurotensin; pl: Isoelectric point; RMT: Receptor-mediated transcytosis; mTfR: Mouse transferrin receptor; TLR4: Toll-like receptor.

\section{Acknowledgements}

We thank Veronique Hendrickx and Jonas Verwaeren for animal husbandry. We would like to thank the VIB Nanobody Core for performing the immunizations and generating the nanobody-displaying phage library.

\section{Authors' contributions}

Design or conceptualization of the study: MD, BDS. Collection, analysis and interpretation of the data: YW, TJ, MD. Drafting and revising the manuscript: YW, MD, BDS. All authors read and approved the final manuscript.

Funding

Yessica Wouters is a Fonds voor Wetenschappelijk Onderzoek (FWO) fellow (1S08516N). Bart De Strooper and Maarten Dewilde are supported by the Cure Alzheimer's Fund and FWO (grant no. S007918N). Work in the De Strooper laboratory was supported by the KU Leuven, VIB, and a Methusalem grant from KU Leuven and the Flemish Government (grant no. 3M140280), the "Geneeskundige Stichting Koningin Elisabeth", the MRC, the Alzheimer Society, and Alzheimer Research UK. Bart De Strooper is holder of the Bax-Vanluffelen Chair for Alzheimer's Disease.

\section{Availability of data and materials}

The datasets used and/or analyzed in the current study are available from the corresponding authors upon reasonable request.

\section{Ethics approval and consent to participate}

All animal experiments were conducted according to protocols approved by the local Ethical Committee of Laboratory Animals of the KU Leuven (governmental license LA1210579, ECD project number 040/2016) following governmental and EU guidelines. Consent to participate is not applicable since no human subjects were involved.

Consent for publication

Not applicable.

Competing interests

The authors declare that they have no competing interests. 


\section{Author details}

${ }^{1}$ VIB Center for Brain \& Disease Research, Campus Gasthuisberg O\&N4, Herestraat 49, box 602, B-3000 Leuven, Belgium. ${ }^{2}$ Laboratory for the Research of Neurodegenerative Diseases, Department of Neurosciences, Leuven Brain Institute (LBI), KU Leuven, B-3000 Leuven, Belgium. ${ }^{3}$ UK Dementia Research Institute, University College London, London, UK. ${ }^{4}$ VIB Discovery Sciences, B-3000 Leuven, Belgium. ${ }^{5}$ Present Address: Laboratory for Therapeutic and Diagnostic Antibodies, Department of Pharmaceutical and Pharmacological Sciences, KU Leuven, B-3000 Leuven, Belgium.

\section{Received: 3 August 2020 Accepted: 8 October 2020}

\section{Published online: 14 October 2020}

\section{References}

1. Almutairi MMA, Gong C, Xu YG, Chang Y, Shi H. Factors controlling permeability of the blood-brain barrier. Cell Mol Life Sci. 2016;73:57-77.

2. Poduslo JF, Curran GL, Berg CT. Macromolecular permeability across the blood-nerve and blood-brain barriers. Proc Natl Acad Sci U S A. 1994;91:5705-9.

3. Demeule $M$, et al. Identification and design of peptides as a new drug delivery system for the brain. J Pharmacol Exp Ther. 2008;324:1064-72.

4. Yu YJ, et al. Therapeutic bispecific antibodies cross the blood-brain barrier in nonhuman primates. Sci Transl Med. 2014;6:261154.

5. Jefferies WA, et al. Transferrin receptor on endothelium of brain capillaries. Nature. 1984;312:162-3

6. Giugliani R, et al. Neurocognitive and somatic stabilization in pediatric patients with severe Mucopolysaccharidosis Type I after 52 weeks of intravenous brain-penetrating insulin receptor antibody-iduronidase fusion protein (valanafusp alpha): an open label phase 1-2 trial. Orphanet J Rare Dis. 2018;13:110.

7. Okuyama T, et al. Iduronate-2-sulfatase with anti-human transferrin receptor antibody for neuropathic mucopolysaccharidosis II: a phase 1/2 trial. Mol Ther. 2019;27:456-64.

8. Pardridge WM. Blood-brain barrier and delivery of protein and gene therapeutics to brain. Front Aging Neurosci. 2019;11:373.

9. Hamers-Casterman C, et al. Naturally occurring antibodies devoid of light chains. Nature. 1993;363:446-8.

10. Gonzalez-Sapienza G, Rossotti MA, Tabares-da Rosa S. Single-domain antibodies as versatile affinity reagents for analytical and diagnostic applications. Front Immunol. 2017;8:977.

11. Muyldermans S. Nanobodies: natural single-domain antibodies. Annu Rev Biochem. 2013;82:775-97.

12. Eichhoff $A M$, et al. Nanobody-enhanced targeting of AAV gene therapy vectors. Mol Ther Methods Clin Dev. 2019;15:211-20.

13. Bannas P, Hambach J, Koch-Nolte F. Nanobodies and nanobody-based human heavy chain antibodies as antitumor therapeutics. Front Immunol. 2017:8:1603.

14. Fang $T$, et al. Remodeling of the tumor microenvironment by a chemokine/anti-PD-L1 nanobody fusion protein. Mol Pharm. 2019;16:2838-44

15. Hu Y, Liu C, Muyldermans S. Nanobody-based delivery systems for diagnosis and targeted tumor therapy. Front Immunol. 2017;8:1442.

16. Elverdi T, Eskazan AE. Caplacizumab as an emerging treatment option for acquired thrombotic thrombocytopenic purpura. Drug Des Devel Ther. 2019;13:1251-8.

17. Rasmussen SGF, et al. Crystal structure of the $\beta 2$ adrenergic receptor-Gs protein complex. Nature. 2011;477:549-55.

18. Oliveira S, Heukers R, Sornkom J, Kok RJ, van Bergen en Henegouwen, P. M. P. . Targeting tumors with nanobodies for cancer imaging and therapy. J Control Release. 2013;172:607-17.

19. Li T, et al. Cell-penetrating anti-GFAP VHH and corresponding fluorescent fusion protein VHH-GFP spontaneously cross the blood-brain barrier and specifically recognize astrocytes: application to brain imaging. FASEB J. 2012;26:3969-79.

20. Haqqani AS, et al. Multiplexed evaluation of serum and CSF pharmacokinetics of brain-targeting single-domain antibodies using a NanoLC-SRMILIS method. Mol Pharm. 2013;10:1542-56.

21. Choi HS, et al. Targeted zwitterionic near-infrared fluorophores for improved optical imaging. Nat Biotechnol. 2013;31:148-53.
22. Li T, et al. Camelid single-domain antibodies: a versatile tool for in vivo imaging of extracellular and intracellular brain targets. J Control Release. 2016:243:1-10.

23. Zhang $W$, et al. Differential expression of receptors mediating receptormediated transcytosis (RMT) in brain microvessels, brain parenchyma and peripheral tissues of the mouse and the human. Fluids Barriers CNS. 2020;17:1-17.

24. Friden PM, et al. Anti-transferrin receptor antibody and antibody-drug conjugates cross the blood-brain barrier. Proc Natl Acad Sci U S A. 1991;88:4771-5.

25. Zhang Y, Pardridge WM. Neuroprotection in transient focal brain ischemia after delayed intravenous administration of brain-derived neurotrophic factor conjugated to a blood-brain barrier drug targeting system. Stroke. 2001;32:1378-84

26. Zhang $Y$, Pardridge WM. Delivery of $\beta$-galactosidase to mouse brain via the blood-brain barrier transferrin receptor. J Pharmacol Exp Ther. 2005;313:1075-81.

27. Yu YJ, et al. Boosting Brain Uptake of a Therapeutic Antibody by Reducing Its Affinity for a Transcytosis Target. Sci Transl Med. 2011;3:84ra44.

28. Boado RJ, Zhang Y, Wang Y, Pardridge WM. Engineering and expression of a chimeric transferrin receptor monoclonal antibody for blood-brain barrier delivery in the mouse. Biotechnol Bioeng. 2009;102:1251-8.

29. Li JY, Sugimura K, Boado RJ, Lee HJ, Zhang C, Duebel S, P. W. M. . Genetically engineered brain drug delivery vectors: Cloning, expression and in vivo application of an anti-transferrin receptor single chain antibodystreptavidin fusion gene and protein. Protein Eng. 1999;12:787-96.

30. Hultqvist G, Syvänen S, Fang XT, Lannfelt L, Sehlin D. Bivalent Brain Shuttle Increases Antibody Uptake by Monovalent Binding to the Transferrin Receptor. Theranostics. 2017;7:308-18.

31. Niewoehner J, et al. Increased Brain Penetration and Potency of a Therapeutic Antibody Using a Monovalent Molecular Shuttle. Neuron. 2014;81:49-60.

32. Karaoglu Hanzatian D, et al. Brain uptake of multivalent and multi-specific DVD-Ig proteins after systemic administration. MAbs. 2018;10:765-77.

33. Kariolis MS, et al. Brain delivery of therapeutic proteins using an Fc fragment blood-brain barrier transport vehicle in mice and monkeys. Sci Transl Med. 2020;12:eaay1359.

34. Ullman J, et al. Brain delivery and efficacy of an intravenously-administered lysosomal enzyme using a blood-brain barrier transport vehicle. Sci Transl Med. 2020;12:eaay1163.

35. Atwal JK, et al. A therapeutic antibody targeting BACE1 inhibits amyloid- $\beta$ production in vivo. Sci Transl Med. 2011;3:84ra43.

36. Wang $M$, Jing T, Wang $X$, Yao D. Beta-secretase/BACE1 promotes APP endocytosis and processing in the endosomes and on cell membrane. Neurosci Lett. 2018;685:63-7.

37. Wu L-P, et al. Crossing the blood-brain-barrier with nanoligand drug carriers self-assembled from a phage display peptide. Nat Commun. 2019;10:4635.

38. Meier SR, et al. Antibody-based In Vivo PET imaging detects amyloid- $\beta$ reduction in alzheimer transgenic mice after BACE-1 inhibition. J Nucl Med. 2018:59:1885-91.

39. Ruderisch N, et al. Potent and selective BACE-1 peptide inhibitors lower brain $A \beta$ levels mediated by brain shuttle transport. EBioMedicine. 2017;24:76-92.

40. Zuchero YJY, et al. Discovery of Novel Blood-Brain Barrier Targets to Enhance Brain Uptake of Therapeutic Antibodies. Neuron. 2016;89:70-82.

41. Fenwick N, Griffin G, Gauthier C. The welfare of animals used in science: how the 'Three Rs' ethic guides improvements. Can Vet J. 2009;50:523-30.

42. St-Gelais F, Jomphe C, Trudeau L-E. The role of neurotensin in central nervous system pathophysiology: what is the evidence? J Psychiatry Neurosci. 2006:31:229-45.

43. Popp E, Schneider A, Vogel P, Teschendorf P, Böttiger BW. Time course of the hypothermic response to continuously administered neurotensin. Neuropeptides. 2007;41:349-54.

44. Rostène W, Kitabgi P, Pélaprat D. Neurotensin receptors . Encycl Biol Chem. 2004;3:32-6.

45. Pardon $\mathrm{E}$, et al. A general protocol for the generation of Nanobodies for structural biology. Nat Protoc. 2014;9:674-93.

46. Conrath $\mathrm{KE}$, et al. Beta-lactamase inhibitors derived from single-domain antibody fragments elicited in the camelidae. Antimicrob Agents Chemother. 2001:45:2807-12. 
47. Kille $S$, et al. Reducing codon redundancy and screening effort of combinatorial protein libraries created by saturation mutagenesis. ACS Synth Biol. 2013;2:83-92.

48. Jimenez-Mateos EM, et al. miRNA expression profile after status epilepticus and hippocampal neuroprotection by targeting miR-132. Am J Pathol. 2011;179:2519.

49. JoVE Science Education Database. Compound Administration I. JoVE. 2020. www.jove.comurl. https://www.jove.com/science-education/10198. Accessed 13 Mar 2020

50. Demeule $\mathrm{M}$, et al. Conjugation of a brain-penetrant peptide with neurotensin provides antinociceptive properties. J Clin Invest. 2014;124:1199-213.

51. Paterson J, Webster $\mathrm{Cl}$. Exploiting transferrin receptor for delivering drugs across the blood-brain barrier. Drug Discov Today Technol. 2016;20:49-52.

52. Boules M, Li Z, Smith K, Fredrickson P, Richelson E. Diverse Roles of Neurotensin Agonists in the Central Nervous System. Front Endocrinol (Lausanne). 2013;4:36.

53. Choi $K$, et al. A novel stroke therapy of pharmacologically induced hypothermia after focal cerebral ischemia in mice. FASEB J. 2012;26:2799-810.

54. Katz LM, Young AS, Frank JE, Wang Y, Park K. Regulated hypothermia reduces brain oxidative stress after hypoxic-ischemia. Brain Res. 2004a;1017:85-91.

55. Katz LM, Young A, Frank JE, Wang Y, Park K. Neurotensin-induced hypothermia improves neurologic outcome after hypoxic-ischemia. Crit Care Med. 2004b;32:806-10.

56. van der Worp HB, Sena ES, Donnan GA, Howells DW, Macleod MR. Hypothermia in animal models of acute ischaemic stroke: a systematic review and meta-analysis. Brain. 2007;130:3063-74.

57. Kleven MD, Jue S, Enns CA. Transferrin receptors TfR 1 and TfR2 bind transferrin through differing mechanisms. Biochemistry. 2018;57:1552-9.

58. Mizutani T, Ishizaka A, Nihei C. Transferrin receptor 1 facilitates poliovirus permeation of mouse brain capillary endothelial cells. J Biol Chem. 2016;291:2829-36.
59. Johnsen KB, et al. Antibody affinity and valency impact brain uptake of transferrin receptor-targeted gold nanoparticles. Theranostics. 2018:8:3416-36.

60. Zimmermann I, et al. Synthetic single domain antibodies for the conformational trapping of membrane proteins. Elife. 2018;7:e34317.

61. Bien-Ly N, et al. Transferrin receptor (TfR) trafficking determines brain uptake of TfR antibody affinity variants. J Exp Med. 2014;211:233-44.

62. Kijanka G, Prokopowicz M, Schellekens H, Brinks V. Influence of aggregation and route of injection on the biodistribution of mouse serum albumin. PLoS ONE. 2014;9:e85281.

63. Sumbria RK, et al. Pharmacokinetics and brain uptake of an IgG-TNF decoy receptor fusion protein following intravenous, intraperitoneal, and subcutaneous administration in mice. Mol Pharm. 2013;10:1425-31.

64. Cortez-Retamozo V, et al. Efficient tumor targeting by single-domain antibody fragments of camels. Int J Cancer. 2002;98:456-62.

65. Nabuurs RJA, et al. In vivo detection of amyloid- $\beta$ deposits using heavy chain antibody fragments in a transgenic mouse model for alzheimer's disease. PLoS ONE. 2012;7:1-10.

66. Keyaerts M, et al. Phase I Study of 68Ga-HER2-Nanobody for PET/ CT Assessment of HER2 Expression in Breast Carcinoma. J Nucl Med. 2016;57:27-33.

67. Rotman M, et al. Fusion of hlgG1-Fc to 111 In-anti-amyloid single domain antibody fragment $\mathrm{VHH}$-pa2H prolongs blood residential time in APP/PS1 mice but does not increase brain uptake. Nucl Med Biol. 2015:42:695-702.

68. Bell A, et al. Differential tumor-targeting abilities of three single-domain antibody formats. Cancer Lett. 2010;289:81-90.

\section{Publisher's Note}

Springer Nature remains neutral with regard to jurisdictional claims in published maps and institutional affiliations.
Ready to submit your research? Choose BMC and benefit from:

- fast, convenient online submission

- thorough peer review by experienced researchers in your field

- rapid publication on acceptance

- support for research data, including large and complex data types

- gold Open Access which fosters wider collaboration and increased citations

- maximum visibility for your research: over $100 \mathrm{M}$ website views per year

At $\mathrm{BMC}$, research is always in progress.

Learn more biomedcentral.com/submissions 